\title{
Health worker led breast examination in rural India using electro-mechanical hand-held breast palpation device
}

\begin{abstract}
Introduction: Breast cancer is the most common female cancer worldwide representing nearly a quarter $(23 \%)$ of all cancers in women. In India, breast cancer is the leading site of cancer. Due to, point of care pre-screening tool operated by community health workers. limitations of resources in India, breast screening is not done at regular intervals and is inaccessible in most parts of the country. The study was conducted with the aim to assess the feasibility of iBreastExam (iBE), a radiation free, painless, portable.
\end{abstract}

Materials and methods: 1300 female subjects, above 30years were enrolled in the study over 6months and underwent both iBE and Clinical Breast Examination.

Results: Out of 1420 women who were approached for the study, 1300(91.5\%) consented for the study, indicating acceptability rate for the technique. 146(11.2\%) women were positive on either or both modalities and underwent breast ultrasonography. 18 patients were found to have significant lesions on USG, for which they underwent breast fine needle aspiration cytology or biopsy and 3 breast cancers were confirmed. All patients who consented for the study, were comfortable taking iBE test due to its radiation free and painless nature.

Conclusions: Breast examination by a radiation-free and painless electro-mechanical hand-held breast palpation device (iBreastExam) is socio-culturally acceptable in the rural environment. The device detected all clinically significant lumps. This study highlights the successful implementation of iBreastExam in the community setting and its potential for mass breast cancer screening in rural environment.
Volume 9 Issue 3 - 2018

Rohan Khandelwal

Consultant - Breast Onco-surgeon,W Pratiksha Hospital, India

Correspondence: Rohan Khandelwal, Consultant - Breast Onco-surgeon, W Pratiksha Hospital, Gurgaon, India, Tel +919810072878, Email rohankhandelwal@gmail.com

Received: April 19, 2018 | Published: June 20, 2018

\section{Introduction}

Breast cancer is the most common female cancer worldwide representing nearly a quarter $(23 \%)$ of all cancers in women. ${ }^{1,2}$ The global burden of breast cancer is expected to cross 2 million by the year 2030, with growing proportions from developing countries. ${ }^{3}$ Although age-standardised incidence rates in India are lower than in the United Kingdom (UK) (25.8 versus 95per 100,000), mortality rates are nearly as high (12.7 versus 17.1 per 100,000 , respectively) as those of the UK. ${ }^{1}$ Breast cancer incidence rates within India display a 3-4-fold variation across the country, with the highest rates observed in the Northeast and in major metropolitan cities such as Mumbai and New Delhi. ${ }^{4}$

Diagnosis at advanced stages of disease contributes to a high mortality rate among women due to breast cancer, which can be attributed to low levels of awareness, ineffective screening methods, cumbersome referral pathways to diagnosis, limited access to effective treatment at regional cancer centres and incomplete treatment regimens. ${ }^{3,5-10}$ With the rising breast cancer incidence in India ${ }^{4}$ and disproportionately higher mortality. ${ }^{11,12}$ it is essential to increase awareness and develop effective screening modalities which can work in large scale settings.

Mammography led screening is virtually impossible in India, with limited radiology manpower and competing healthcare priorities. While CBE (CBE) is affordable (Int $\$ 793$ per life year saved), its effectiveness is limited (3). Also, training health workers to perform consistently high quality breast examination is challenging (4).

In LMICs, community health workers (CHWs) often represent an affordable resource for the education and provision of preventive, primary and promotive healthcare. Low-cost, user-friendly technology can help equip minimally trained CHWs to administer standardized breast exams without any special infrastructure. The low-cost technology must perform with better detection sensitivity than CBE (higher than 50\%) and equally high specificity as CBE (94\%) to accurately and effectively identify breast lumps in need of further diagnostic follow-up (diagnostic ultrasound, breast biopsy) without clogging the under-resourced infrastructure with false positives due to typical benign breast features (tissue variability, lumpiness and nodularity).

With this background, a study was designed to assess the feasibility of iBreastExam (iBE) in a community setting with the help of existing healthcare resources such as community health workers (CHWs) and social workers

\section{Materials and methods}

Out of 1420 women who were approached for the study, $1300(91.5 \%)$ consented for the study, indicating acceptability rate for the technique. These 1300 women above 30 years of age were enrolled in the study over a period of 6months. Female community health 
workers from Aastha Breast Cancer Support Group and Lions Club (Pune, India) were trained to operate iBE device to perform bilateral breast examinations.

Informed consent was taken in the local language before enrolling the patients for this study. Subjects were not offered any incentive for participating in the study. Subjects were asked to lay on a medical bed, after privately disrobing from the waist up. The operator performed the $\mathrm{iBE}$ in a clock-wise manner.

iBE comprises of an array of piezoelectric sensors that electronically palpate the breast and can differentiate between varying tissue elasticity. The "electronic palpation" was entirely automated and controlled internally. Following the iBE exam, patients underwent a routine Clinical Breast Examination $(\mathrm{CBE})$ by an experienced doctor. The doctor was unaware of the iBE findings while performing the CBE. Patients who were either positive on iBE, CBE or both, underwent a breast ultrasonography exam (USG). If USG test was positive, patients were subjected to a breast FNAC or biopsy. IRB \& Ethical committee clearance for this study were taken at Sasur Hospital in Pune.

\section{Results}

1300 subjects were enrolled in the study over 6months. The average age was 42.37 years. $\mathrm{iBE}$ and Expert CBE was done for all patients.

Out of the 1300 subjects, $\mathrm{iBE}$ and Expert CBE were both negative in $1,139(87.6 \%)$ women. In 161(12.4\%) women, either iBE or Expert $\mathrm{CBE}$ or both were positive. iBE was positive in $130(10 \%)$ women and Expert CBE was positive in 101(7.7\%) women. 15 women from this group, refused to obtain a follow-up breast USG. USG was done for women either positive on $\mathrm{iBE}, \mathrm{CBE}$ or both.

Of the 130 women that were positive on $\mathrm{iBE}, 103$ cases were confirmed by breast USG and 27 cases did not show any signs of breast abnormalities as per breast USG. Of the 138 women that were positive on CBE, 101 cases were confirmed by breast USG and 37 cases did not show any signs of breast abnormalities as per breast USG.

Of the total 146 women positive (excluding the ones that refused follow-up), breast USG was positive in 101(69.2\%) women for clinically relevant breast lesions. Of these, 18 women were recommended breast FNAC/biopsy and $3(0.23 \%)$ cases were diagnosed positive for breast cancer (Table 1-4).

Table I

\begin{tabular}{ll} 
Total number of women screened & 1300 \\
Normal women (iBE \& CBE negative) & 1139 \\
iBE positive & 130 \\
CBE positive & 138 \\
Positive findings on USG & 101 \\
\hline
\end{tabular}

Table 2

\begin{tabular}{lll}
\hline \multicolumn{3}{l}{ Comparison of iBE to expert CBE } \\
\hline & CBE negative & CBE positive \\
\hline iBE Negative & 1139 & 0 \\
iBE Positive & 2 & 101 \\
\hline
\end{tabular}

Table 3

\begin{tabular}{|c|c|c|}
\hline \multicolumn{3}{|c|}{$\begin{array}{l}\text { Comparison of iBE to breast USG (truth) for breast lesic } \\
\text { detection }\end{array}$} \\
\hline & USG negative & USG positive \\
\hline iBE Negative & $\mathrm{N} / \mathrm{A}$ & N/A \\
\hline ¡BE Positive & 27 & 103 \\
\hline
\end{tabular}

Table 4

\begin{tabular}{lll}
$\begin{array}{l}\text { Comparison of CBE to breast USG (truth) for breast lesion } \\
\text { detection }\end{array}$ \\
\hline & USG negative & USG positive \\
\hline CBE Negative & N/A & N/A \\
CBE Positive & 37 & 101 \\
\hline
\end{tabular}

\section{Discussion}

Electro-mechanical hand-held breast palpation device (iBreast Exam) uses piezoelectric tactile sensor invented at Drexel University. The patented ceramic sensor can assess the differences in tissue elasticity from the surface of the breast, non-invasively by creating micro-palpations that are driven from within the sensor. Using direct/ reverse piezoelectric effects, the piezoelectric sensor array enables "electronic palpation", from within the sensor and independent of the community health worker. And hence, in real-time, the sensor can assess the changes in the elastic modulus between normal breast tissue and abnormal masses that are stiffer.

The device (Figures 1A-D) has two major components; a scanner probe with multiple piezoelectric tactile pressure sensors and a computer tablet with proprietary software. The iBE device is battery powered and wireless; the software in the tablet displays compression data recordings. At the end of the test, a report is generated instantly showing normal areas in green colour and abnormal areas in red colour.

This study was conducted in rural environment where it was not feasible to provide standard of care imaging tests to every participant. For this reason, women that were negative on both $\mathrm{iBE}$ and $\mathrm{CBE}$ were not followed-up by any other imaging modality.

With these limitations, this study was primarily designed to validate the feasibility of $\mathrm{iBE}$ in rural settings and it's ability to identify clinically relevant breast lumps and possibly breast cancer, in asymptomatic women in rural areas.

Expert CBE is known to have high specificity (92-94\%), meaning low false-positive rate. In the study, there were 2 cases that were positive on $\mathrm{iBE}$ and negative on $\mathrm{CBE}$, while there were 1,139 cases that were negative on both tests. In this regard, $\mathrm{BBE}$ demonstrated a low false positive rate, comparable to that of Expert CBE.

Screening mammograms in USA have shown sensitivity as low as $54 \%$ to detect invasive breast cancer, especially if the women are young and have dense breasts. ${ }^{4-6}$ Clinical study by Broach et al reported iBreastExam sensitivity of $86 \%$ and specificity of $89 \%$ to detect breast masses as compared to standard of care imaging tests such as mammography and breast ultrasound. ${ }^{13,14}$ Study by Somashekhar et al reported sensitivity of $85 \%$ and specificity of $93 \%$ to detect breast lesions in a setting where iBE was performed blinded 
to standard imaging tests. ${ }^{15}$ iBreastExam has reported comparable performance characteristics to standard of care imaging viz. screening mammography.

iBE was well accepted and used with comfort by the women in the study. It was found that it is easier to convince women to take iBE test against mammography on account of pain and radiation associated with mammography. Clinical breast exam is a subjective manual test in comparison with $\mathrm{iBE}$, which is a standardized device based test which eliminates human subjectivity while performing breast examinations.

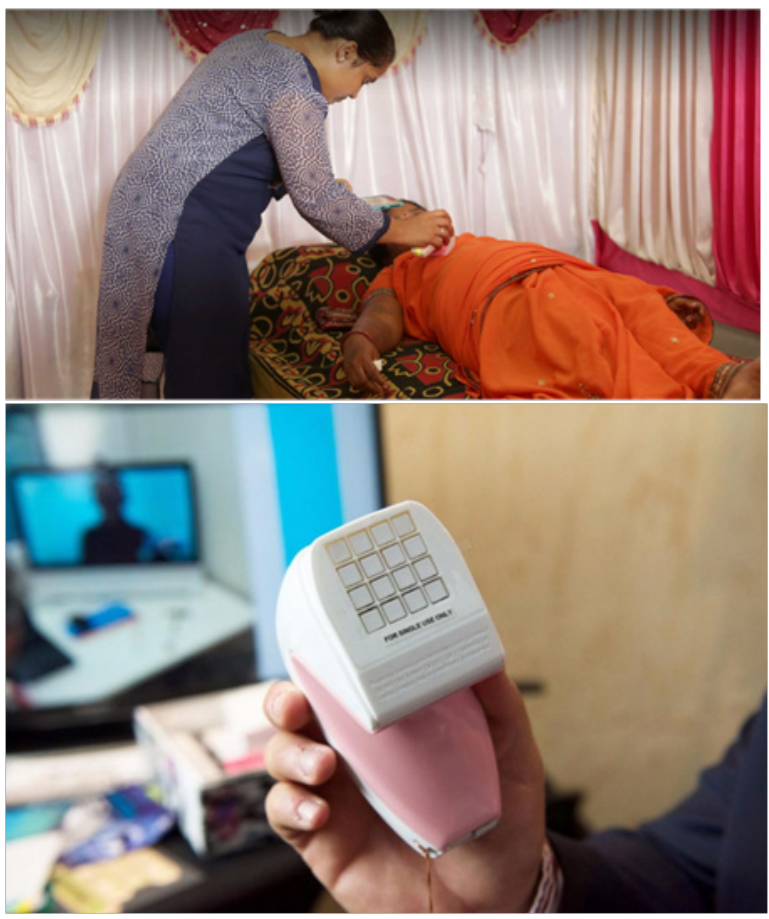

\section{Figure I}

A) iBE device in use.

B) The iBE Scanner Probe.

C) Data pressure map illustration of normal or negative results.

D) Data pressure map illustration of abnormal or positive results.

\section{Conclusion}

As a hand-held, radiation-free, painless and easy to use electromechanical tool, iBreastExam can potentially be a powerful screening tool for use in the developing world with limited resources where mammography and CBE by a trained physician is not readily available. From care pathway standpoint, Positive findings by the iBE can allow for triaged patients to be examined by targeted breast ultrasound at primary care level and before referring them to a larger medical centres where FNAC/biopsy as well as surgical excision could be performed. Training lay people on the use of the device could potentially provide screening to rural and underserved areas of underdeveloped nations where women historically have had no access to breast cancer early detection.

The iBE device is currently being piloted in a rural area in India where due to limited economic resources, women have essentially no access to mammography and present with late-stage breast cancers. As described in the literature, late-stage breast cancers have poorer prognosis than early stage breast cancers. ${ }^{16}$ Due to the significantly lower cost of the iBE compared to conventional mammography, we see this device as a very useful adjunct to breast cancer detection worldwide in low- and-middle income countries (LMICs) with limited medical economic resources and a need for improved breast cancer early diagnosis.

\section{Acknowledgements}

None.

\section{Conflict of interest}

Author declares that there is no conflict of interest.

\section{References}

1. Ferlay J, Soerjomataram I, Ervik M. Estimated Cancer Incidence, Mortality and Prevalance Worldwide in 2012. 2012.

2. Global Burden of Disease Cancer Collaboration, The global burden of cancer 2013. JAMA Oncol. 2015 1(4):505-27.

3. Jemal A, Bray F, Melissa MC, et al. Global cancer statistics. CA Cancer J Clin. 2011;61(2):69-90.

4. National Cancer Registry Programme. National Centre for Disease Informatics and Research. Indian Council of Medical Research., Three year report of population based cancer registries 2009-2011 national cancer registry programme. National Cancer Registry. 2013.

5. Rosenberg RD, Hunt WC, Williamson MR, et al. Effects of age, breast density, ethnicity, and estrogen replacement therapy on screening mammographic sensitivity and cancer stage at diagnosis: review of 183,134 screening mammograms in Albuquerque, New Mexico. Radiology. 1998;209(2):511-8.

6. Kerlikowske K, Grady D, Barclay J, et al. Likelihood ratios for modern screening mammography. Risk of breast cancer based on age and mammographic interpretation. JAMA. 1996;276(1):39-43.

7. Zhang Z. Detection of Breast Cancer With Addition of Annual Screening Ultrasound or a Single Screening MRI to Mammography in Women With Elevated Breast Cancer Risk. JAMA, 2012;307(13):1394-404.

8. Sharma K, Costas A, Shulman LN, et al. A systematic review of barriers to breast cancer care in developing countries resulting in delayed patient presentation. J Oncol. 2012;2012.

9. Jones S, Johnson K. Women's awareness of cancer symptoms: a review of the literature. Women's Health. 2012;8(5):579-591.

10. Jones C, Maben J, Jack RH, et al. A systematic review of barriers to early presentation and diagnosis with breast cancer among black women. $B M J$ Open. 2014;4(2).

11. Dey S. Preventing breast cancer in LMICs via screening and/or early detection: the real and the surreal. World J Clin Oncol. 2014;5(3):509519 .

12. Dikshit R, Gupta PC, Ramasundarahettige $C$, et al. Cancer mortality in India: a nationally representative survey. Lancet. 2012;379(9828):18071816.

13. Broach RB, Geha R, Englander BS, et al. A cost-effective handheld breast scanner for use in low-resource environments: a validation study. World Journal of Surgical Oncology. 2016;14:277. 
14. Broach, Robyn B. "A Cost-Effective Handheld Breast Scanner for Use in Low-Resource Environments: a Validation Study." World Journal of Surgical Oncology, BioMed Central. 2016.

15. Somashekhar SP, Vijay R, Ananthasivan R, et al. Noninvasive and lowcost technique for early detection of clinically relevant breast lesions using a handheld point-of-care medical device (iBE): prospective three-arm triple-blinded comparative study. Indian $J$ Gynecol Oncol. 2016;14:26.

16. Torre L, Bray F, Siegel RL, et al. Global cancer statistics, 2012. CA Cancer J Clin. 2015;65(2):87-108. 